\title{
A Lagrangian approach to classical thermodynamics
}

DOI:

$10.1209 / 0295-5075 / 117 / 30002$

\section{Document Version}

Accepted author manuscript

Link to publication record in Manchester Research Explorer

\section{Citation for published version (APA):}

Stokes, A. (2017). A Lagrangian approach to classical thermodynamics. EPL, 117(3), [30002]. https://doi.org/10.1209/0295-5075/117/30002

\section{Published in:}

EPL

\section{Citing this paper}

Please note that where the full-text provided on Manchester Research Explorer is the Author Accepted Manuscript or Proof version this may differ from the final Published version. If citing, it is advised that you check and use the publisher's definitive version.

\section{General rights}

Copyright and moral rights for the publications made accessible in the Research Explorer are retained by the authors and/or other copyright owners and it is a condition of accessing publications that users recognise and abide by the legal requirements associated with these rights.

\section{Takedown policy}

If you believe that this document breaches copyright please refer to the University of Manchester's Takedown Procedures [http://man.ac.uk/04Y6Bo] or contact uml.scholarlycommunications@manchester.ac.uk providing relevant details, so we can investigate your claim.

\section{OPEN ACCESS}




\title{
A Lagrangian approach to thermodynamics
}

\author{
Adam Stokes* \\ The Photon Science Institute, University of Manchester, Manchester, M13 9PL, United Kingdom
}

\begin{abstract}
The specification of microstates of interacting dynamical systems is different in Lagrangian and Hamiltonian approaches whenever the interaction Lagrangian depends on generalised velocities. In most all cases of physical interest however, velocity-dependent interaction Lagrangians do not couple velocities belonging to different subsystems. For these cases we define reduced system and bath Lagrangian macrostates, which like the underlying microstates differ from their Hamiltonian counterparts. We then derive exact first and second laws of thermodynamics without any modification of the original system and bath quantities. This approach yields manifestly gauge-invariant definitions of work and free energy, and a gauge-invariant Jarzynski equality is derived. The formalism is applied in deriving the thermodynamic laws for a material system within the radiative reservoir. The Lagrangian partition of the total energy is manifestly gauge-invariant and is in accordance with Poynting's theorem.
\end{abstract}

PACS numbers:

Introduction - The first law of thermodynamics states that the energy change of a system in contact with a heat bath can be partitioned as $\Delta E=Q+W$ where $Q$ denotes the heat absorbed from the bath, and $W$ denotes the work performed on the system. The second law states that the entropy change within the system can be partitioned as $\Delta S_{s}=\Delta_{i} S+\beta Q$ where $\Delta_{i} S \geq 0$ is called the entropy production, and $\beta^{-1}$ is the temperature of the bath. Recent years have seen exciting developments in both statistical mechanics and thermodynamics, which build upon the first and second laws. The most notable of these results are the work and fluctuation theorems [1-6]. As these thermodynamic results continue to be confirmed in experiments, their validity becomes increasingly well-established [7-12]

Somewhat surprisingly, microscopic derivations of the fundamental laws of thermodynamics are scarce. The main reason for this is that it is unclear how to reconcile the presence of a finite system-bath interaction with the additive structure of the laws themselves [13]. This conundrum seems endemic to a Hamiltonian approach, in which the Hamiltonian is partitioned as $H=$ $H_{s}+H_{b}+H_{i}$, where $H_{s}$ and $H_{b}$ refer to the system and bath respectively, and $H_{i}$ describes their interaction. The system and the bath must effectively share the energy in the term $H_{i}$, and it is not obvious whether this energy should be viewed as belonging to the system or to the bath [2].

When the system-bath coupling is sufficiently weak the interaction term $H_{i}$ can be neglected judiciously in order that the thermodynamic laws can be derived while assuming that $H_{s}$ and $H_{b}$ represent the system and bath energies [14-17]. The problem of additivity is therefore sharpest in the strong-coupling regime, which has received considerable attention $[2,4,13]$. Some authors have even suggested that if the coupling is strong enough,

*Electronic address: adam.stokes@manchester.ac.uk the second law can be violated [18-22]

If one seeks to derive the laws of thermodynamics in complete generality then it is evident that some modification of quantities referring to the system must be made within a Hamiltonian approach [13, 16, 23-25]. Recently a general formulation of thermodynamics has been given along these lines by Seifert [13], who employed the potential of mean force concept $[2,13,23,26,27]$. Drawbacks of this approach are that the expressions obtained are somewhat unwieldy, and difficulties arise regarding quantisation [13]. There are presently few alternatives to the approach presented in [13]. One notable alternative has been given by Esposito et al. [16, 28] who derived general first and second laws at the expense of including the interaction Hamiltonian within the system energy. The total energy is then extensive, but the averages of system observables cannot be found using the reduced system state.

In general the task of assigning thermodynamic quantities to the system and to the bath is a difficult one. Previous attempts to do this have been based on a Hamiltonian approach $[13,16]$. When the Hamiltonian and Lagrangian microstates of system and bath coincide a Lagrangian based approach would be equivalent, as is the case for a Brownian particle for example. Yet, for fundamental interactions whereby forces are supplied by gauge fields the specification of microstates differs in Hamiltonian and Lagrangian approaches. In these cases the interaction Lagrangian depends on generalised velocities.

It is specifically the cases in which the Hamiltonian and Lagrangian approaches are different that we focus on here. In such cases the Lagrangian can be partitioned as $L=L_{s}+L_{b}+L_{i}$, such that the total energy is extensive; $E=E_{s}+E_{b}$. A natural identification of the basic thermodynamic quantities is then possible, and the laws of thermodynamics can be derived without approximation. This is achieved without any modification of the quantities that are taken to refer to the "system" and to the "bath". The averages of system observables, including energy, heat, and work, can nevertheless be 
found using a reduced system state. All thermodynamic entropies are of the usual Shannon-von Neumann type familiar from information theory. Thus, an especially simple theory of Lagrangian thermodynamics emerges, which differs from all previously given approaches based on Hamiltonian microstates $[13,15,16]$.

We begin by introducing the theoretical framework employed. We then derive the first and second laws, along with the Jarzynski equality and a Clausius inequality, within the Lagrangian framework. The physical example in which the thermodynamic system and bath consist of charged particles is then presented. Next we discuss how the Lagrangian definitions relate to gauge freedom within the Hamiltonian definition of work, which has been debated extensively in the literature [29-39]. Finally, we summarise our findings.

Model - The state space of the thermodynamic system and thermal bath is taken to be a (possibly infinitedimensional) symplectic Hilbert space $\Omega$. We specify a basis $b_{L}$ of $\Omega$ in which vector components are assumed to represent generalised coordinates and velocities. The coordinates describing the system are denoted $x_{s}^{i}=\left(q^{i}, v^{i}\right)$, while those describing the bath are denoted $x_{b}^{\mu}=\left(Q^{\mu}, V^{\mu}\right)$. Collections of components are denoted with boldface, for example, when $i$ is a discrete index we can write $\mathbf{x}_{s}:=\left(x_{s}^{1}, x_{s}^{2}, \ldots\right)$. In the case of field systems the indices $i, \mu$ vary continuously. The "dot product" notation - must then be understood as denoting an inner-product between fields, and observable averages must be understood using the general theory of integration in Hilbert space [40]. The pair $\mathbf{x}=\left(\mathbf{x}_{s}, \mathbf{x}_{b}\right)$ represents a state of the system-bath composite. The basis $b_{L}$ gives a division of the state space into system and bath state spaces as $\Omega[\mathbf{x}]=\Omega_{s}\left[\mathbf{x}_{s}\right] \oplus \Omega_{b}\left[\mathbf{x}_{b}\right]$. The Lagrangian $L: \Omega \times \mathbb{R} \rightarrow \mathbb{R}$ describing the system and bath can be viewed as a function of components having the form

$$
L=L_{s}\left(\mathbf{x}_{s}, \lambda\right)+L_{i}(\mathbf{x}, \lambda)+L_{b}\left(\mathbf{x}_{b}\right)
$$

where $L_{s}\left(L_{b}\right)$ depends only on the system (bath) state, and $L_{i}$ describes the system-bath interaction. We have allowed for the possibility that the system is externally driven by including a possible dependence of $L$ on a control parameter $\lambda$.

We assume that the total system also admits a Hamiltonian description. Canonical momenta are defined via a Legendre transformation as $p^{i}:=\partial L / \partial v^{i}$ and $P^{\mu}:=$ $\partial L / \partial V^{\mu}$. The Legendre transformation induces a basis transformation $b_{L} \rightarrow b_{H}$ within $\Omega$. A vector with components $\mathbf{x}$ in the basis $b_{L}$ has components $\mathbf{y}$ in the basis $b_{H}$, where $\mathbf{y}=\left(\mathbf{y}_{s}, \mathbf{y}_{b}\right)$ with $y_{s}^{i}=\left(q^{i}, p^{i}\right)$ and $y_{b}^{\mu}=\left(Q^{\mu}, P^{\mu}\right)$. The Jacobian matrix of the invertible coordinate transformation $\mathbf{x} \leftrightarrow \mathbf{y}$ is denoted $J$. By assumption $|J| \neq 0$, which implies that the space $\Omega$ possesses a canonical symplectic structure $\omega$. The basis $b_{H}$ gives a second division of the state space as $\Omega[\mathbf{y}]=\Omega_{s}\left[\mathbf{y}_{s}\right] \oplus \Omega\left[\mathbf{y}_{b}\right]$. It is important to recognise that this division is not equivalent to that given by $b_{L}$, because owing to the interaction term in (1) the canonical momenta of the system $p^{i}$ generally depend on $\mathbf{x}_{b}$. Similarly, the $P^{\mu}$ depend on $\mathbf{x}_{s}$ in general. In other words the bases $b_{L}$ and $b_{H}$ give different specifications of what constitute the "system" and "bath".

The dynamics of the total system trace a path in $\Omega$ with symplectic flow $F_{t}: \Omega \rightarrow \Omega$. On the component level the flow maps an initial point $\mathbf{y}$ to a point $F_{t}(\mathbf{y})=\mathbf{y}_{t}$ at time $t>0$. An observable $f: \Omega \times \mathbb{R} \rightarrow \mathbb{R}$ can be viewed as a function $f_{L}(\mathbf{x}, \lambda)$ of positions and velocities, and also as a function $f_{H}(\mathbf{y}, \lambda)=f_{L}(\mathbf{x}(\mathbf{y}), \lambda)$ of positions and momenta. We define a time-dependent probability distribution with density $\rho$ such that the average of an observable $f$ at time $t$ is

$$
\langle f\rangle_{t}=\int d \mathbf{y} \rho(\mathbf{y}, 0) f_{H}\left(\mathbf{y}_{t}(\mathbf{y}), \lambda_{t}\right)
$$

where $\rho(\mathbf{y}, 0)$ is the density at the initial time $t=0$. The density satisfies $d \rho\left(\mathbf{y}_{t}, t\right) / d t=0$, so that $\rho(\mathbf{y}, t)$ satisfies Liouville's equation viewed as a partial differential equation in $\mathbf{y}$ and $t$. Using these results or by noting that $F_{t}$ is symplectic one finds that the invertible transformation $\mathbf{y} \leftrightarrow \mathbf{y}_{t}$ has Jacobian one (Liouville's theorem). Eq. (2) can therefore be written

$$
\langle f\rangle_{t}=\int d \mathbf{y} \rho(\mathbf{y}, t) f_{H}\left(\mathbf{y}, \lambda_{t}\right)
$$

Eqs. (2) and (3) define the average of an observable viewed as a function of positions and momenta $\mathbf{y}$. In the Lagrangian approach the specification of "system" and "bath" is made in terms of the positions and velocities $\mathbf{x}$. Viewed as a function $f_{L}$ of $\mathbf{x}$ the average of $f$ can be written

$$
\begin{aligned}
\langle f\rangle_{t} & =\int d \mathbf{x}|J| \rho_{L}(\mathbf{x}, 0) f_{L}\left(\mathbf{x}_{t}(\mathbf{x}), \lambda_{t}\right) \\
& =\int d \mathbf{x}|J| \rho_{L}(\mathbf{x}, t) f_{L}\left(\mathbf{x}, \lambda_{t}\right)
\end{aligned}
$$

where $\rho_{L}(\mathbf{x}, t):=\rho(\mathbf{y}(\mathbf{x}), t)$.

Here we are interested in situations for which Lagrangian and Hamiltonian microstates are necessarily different, which occurs for velocity-dependent interaction Lagrangians. Since Lagrangians that couple different velocities tend not to occur in nature, the case of most physical significance is that in which $L_{i}$ is linear in the generalised velocities

$$
L_{i}=\mathbf{v} \cdot \mathbf{f}(\mathbf{q}, \mathbf{Q})+\mathbf{V} \cdot \mathbf{g}(\mathbf{q}, \mathbf{Q})
$$

where $\mathbf{f}$ and $\mathbf{g}$ are arbitrary. Eq. (5) implies that the total energy of the system and bath is an extensive quantity;

$$
\begin{aligned}
E(\mathbf{x}, \lambda) & =\left[\mathbf{v} \cdot \frac{\partial L_{s}(\lambda)}{\partial \mathbf{v}}-L_{s}(\lambda)\right]+\left[\mathbf{V} \cdot \frac{\partial L_{b}}{\partial \mathbf{V}}-L_{b}\right] \\
& \equiv E_{s}\left(\mathbf{x}_{s}, \lambda\right)+E_{b}\left(\mathbf{x}_{b}\right)
\end{aligned}
$$

A significant example of the form in eq. (6) is found in the interaction between matter and transverse radiation. When expressed in terms of $\mathbf{y}$ the energy becomes the Hamiltonian $H(\mathbf{y})$. 
An important consequence of eq. (5) is that the Jacobian matrix $J$ has the block diagonal form $J=$ $\operatorname{diag}\left(J_{s}, J_{b}\right)$ where $J_{s}^{i j}=\partial^{2} L_{s} / \partial v^{i} \partial v^{j}$ and $J_{b}^{\mu \nu}=$ $\partial^{2} L_{b} / \partial V^{\mu} \partial V^{\nu}$. The determinant $|J|$ therefore factorises as $|J|=\left|J_{s}\right|\left|J_{b}\right|$. This allows us to define reduced system and bath probability distributions with associated densities $\rho_{L s}$ and $\rho_{L b}$ given by

$$
\rho_{L s, b}\left(\mathbf{x}_{s, b}, t\right)=\int d \mathbf{x}_{b, s}\left|J_{b, s}\right| \rho_{L}(\mathbf{x}, t)
$$

which satsify the normalisation conditions

$$
\int d \mathbf{x}_{s}\left|J_{s}\right| \rho_{L s}\left(\mathbf{x}_{s}, t\right)=1=\int d \mathbf{x}_{b}\left|J_{b}\right| \rho_{L b}\left(\mathbf{x}_{b}, t\right)
$$

In the Lagrangian approach system observables at time $t$ have the form $f_{L s}\left(\mathbf{x}_{s}, \lambda_{t}\right)$. The average of such an observable is

$$
\left\langle f_{s}\right\rangle_{t}=\int d \mathbf{x}_{s}\left|J_{s}\right| \rho_{L s}\left(\mathbf{x}_{s}, t\right) f_{L s}\left(\mathbf{x}_{s}, \lambda_{t}\right)
$$

Thermodynamics - The specification of the basic thermodynamic quantities and the derivation of the thermodynamic laws now follows with relative ease. The energy of the system and bath are already given in (6) and the extensive form ensures that all sources and sinks of energy are properly accounted for [41-46]. The change in energy of the total system is equal to the work performed on the system;

$$
w=E\left(\mathbf{x}_{t}, \lambda_{t}\right)-E\left(\mathbf{x}_{0}, \lambda_{0}\right)=\int_{0}^{t} d t^{\prime} \dot{\lambda}_{t^{\prime}} \frac{\partial E_{s}\left(\mathbf{x}_{s, t^{\prime}}, \lambda_{t^{\prime}}\right)}{\partial \lambda_{t^{\prime}}} .
$$

The heat absorbed from the bath is

$$
q=\int_{0}^{t} d t^{\prime} \dot{\mathbf{x}}_{s, t^{\prime}} \cdot \frac{\partial E_{s}\left(\mathbf{x}_{s, t^{\prime}}, \lambda_{t^{\prime}}\right)}{\partial \mathbf{x}_{s, t^{\prime}}}
$$

The first law $\Delta E_{s}=q+w$ is therefore satisfied on the trajectory level. By averaging this expression one obtains the corresponding first law on the ensemble level. In the Lagrangian approach the heat absorbed by the system is automatically the energy lost by the heat bath, $q=-\Delta E_{b}$. Furthermore, using the right-hand-side of eq. (4) the average heat $Q:=\langle q\rangle_{t}$ can be found using the reduced system density $\rho_{L s}\left(\mathbf{x}_{s}, t\right)$, without any modification of the original system or bath quantities.

In order to derive the second law we define the entropy in the usual form

$$
S=-\int d \mathbf{x}|J| \rho_{L}(\mathbf{x}, t) \ln \rho_{L}(\mathbf{x}, t)=-\left\langle\ln \rho_{L}\right\rangle_{t}
$$

Instantaneous system and bath partition functions are defined as $Z_{s, b}:=\int d \mathbf{x}_{s, b}\left|J_{s, b}\right| e^{-\beta E_{s, b}}$. If we assume an initially equilibrated total system, or more generally we assume $\rho_{L}(\mathbf{x}, 0)=\rho_{L s}\left(\mathbf{x}_{s}\right) e^{-\beta E_{b}} / Z_{b}$ in which the system density $\rho_{L s}\left(\mathbf{x}_{s}\right)$ is arbitrary, a few lines of calculation yields the change in system entropy as

$$
\begin{aligned}
\Delta S_{s}(t)= & \int d \mathbf{x}_{s}\left|J_{s}\right|\left[\rho_{L s}\left(\mathbf{x}_{s}, t\right) \ln \rho_{L s}\left(\mathbf{x}_{s}, t\right)\right. \\
& \left.-\rho_{L s}\left(\mathbf{x}_{s}, 0\right) \ln \rho_{L s}\left(\mathbf{x}_{s}, 0\right)\right]=\Delta_{i} S+\beta Q
\end{aligned}
$$

where

$$
\Delta_{i} S=\int d \mathbf{x}|J| \rho_{L}(\mathbf{x}, t) \ln \left[\frac{\rho_{L}(\mathbf{x}, t) Z_{b}}{\rho_{L s}\left(\mathbf{x}_{s}\right) e^{-\beta E_{b}\left(\mathbf{x}_{b}\right)}}\right] \geq 0
$$

is a non-negative entropy production. We have therefore established the first and second laws of thermodynamics within the Lagrangian framework.

Further results can be derived along similar lines. For example, assuming an initially equilibrated total system, using eq. (10) we easily obtain Jarzynski's equality

$$
\left\langle e^{-\beta w}\right\rangle_{t}=e^{-\beta \Delta F_{s}^{e q}}
$$

where

$$
\Delta F_{s}^{e q}=-\frac{1}{\beta} \ln \left[\frac{Z_{s}^{t}}{Z_{s}^{0}}\right], \quad Z_{s}^{t}:=\int d \mathbf{x}_{s}\left|J_{s}\right| e^{-\beta E_{s}\left(\mathbf{x}_{s}, \lambda_{t}\right)} .
$$

The equilibrium free energy difference in (15) refers to the system alone without any modification of the system quantities $[2,13]$, and without an assumption of weakcoupling [1].

A fundamental expression that can be obtained easily within the Lagrangian approach, but cannot be obtained in full generality in any obvious way within the Hamiltonian approach, is

$$
\left(\beta_{s}-\beta_{b}\right) Q=\Delta_{i} S+\beta_{s} \Delta F_{s} \geq 0,
$$

where we have assumed $\rho_{L}(0, \mathbf{x})=e^{-\beta_{s} E_{s}-\beta_{b} E_{b}} / Z_{s} Z_{b}$ and that the system is not driven $\Delta E_{s}=q$. The non-equilibrium free energy in (17) is defined as $F_{s}=$ $\left\langle E_{s}\right\rangle-\beta_{s}^{-1} S_{s}$. An implication of (17) is that $\operatorname{sgn} Q=$ $\operatorname{sgn}\left(\beta_{b}^{-!}-\beta_{s}^{-1}\right)$, which is a concise expression of Clausius' statement; heat will not spontaneously flow from a hotter to a colder system [47]. The proof of (17) uses the identity

$$
\Delta F_{s}=\int d \mathbf{x}_{s}\left|J_{s}\right| \rho_{L s}\left(\mathbf{x}_{s}, t\right) \ln \left[\frac{\rho_{L s}\left(\mathbf{x}_{s}, t\right)}{\rho^{e q}\left(\mathbf{x}_{\mathbf{s}}\right)}\right] \geq 0,
$$

which expresses the free energy change as a relative entropy. Inequality (17) is obtained without assuming weak-coupling [48] and without any restriction to thermal operations [43].

Example - We consider point charges $e_{\alpha}$ in the radiation reservoir, which is a paradigmatic example within the open systems approach to thermodynamics [15, 49]. In an open systems treatment based on the Hamiltonian formalism various approximations are made, which are justified on the grounds of weak light-matter coupling $[14,15,17,49]$. No approximations will be made here. 
The particles are driven by external potentials $\phi_{e}(\lambda), \mathbf{A}_{e}(\lambda)$ describing the influence of the external agent. When the bath is purely electromagnetic it is possible to include the inter-particle coulomb energies within the system energy. The bath is described by the gauge-invariant transverse potential $\mathbf{A}_{\mathrm{T}}$ and associated transverse electric and magnetic fields $\mathbf{E}_{\mathrm{T}}=-\dot{\mathbf{A}}_{\mathrm{T}}$ and $\mathbf{B}=\nabla \times \mathbf{A}_{\mathrm{T}}$. The system charge and current densities are $\rho(\mathbf{x})=\sum_{\alpha} e_{\alpha} \delta\left(\mathbf{x}-\mathbf{r}_{\alpha}\right)$ and $\mathbf{J}(\mathbf{x})=\sum_{\alpha} e_{\alpha} \dot{\mathbf{r}}_{\alpha} \delta\left(\mathbf{x}-\mathbf{r}_{\alpha}\right)$. The total energy of the system is $E=E_{s}+E_{b}$ where

$$
\begin{aligned}
& E_{s}=\sum_{\alpha} \frac{1}{2} m \dot{\mathbf{r}}_{\alpha}^{2}+V\left(\lambda_{t}\right), \\
& E_{b}=\frac{1}{2} \int d^{3} x\left[\mathbf{E}_{\mathrm{T}}^{2}+\mathbf{B}^{2}\right] .
\end{aligned}
$$

where $V\left(\lambda_{t}\right)=\sum_{\alpha} e_{\alpha} \phi_{e}\left(\mathbf{r}_{\alpha}, \lambda_{t}\right)+\sum_{\beta \neq \alpha} U\left(\mathbf{r}_{\beta}, \mathbf{r}_{\alpha}\right)$ and $U$ denotes the inter-particle Coulomb energies. The energy coincides with the Hamiltonian when written in terms of the particle and field canonical momenta conjugate to $\mathbf{r}_{\alpha}$ and $\mathbf{A}_{\mathrm{T}}$, which are

$$
\begin{aligned}
\mathbf{p}_{\alpha} & =m \dot{\mathbf{r}}_{\alpha}+e_{\alpha} \mathbf{A}_{\mathrm{T}}\left(\mathbf{r}_{\alpha}\right)+e_{\alpha} \mathbf{A}_{e}\left(\mathbf{r}_{\alpha}, \lambda_{t}\right), \\
\boldsymbol{\Pi}_{\mathrm{T}}(\mathbf{x}) & =-\mathbf{E}_{\mathrm{T}}(\mathbf{x}) .
\end{aligned}
$$

Note that the particle canonical momenta $\mathbf{p}_{\alpha}$ do not coincide with the manifestly gauge-invariant mechanical momenta $m \dot{\mathbf{r}}_{\alpha}$. Unlike the microstates $\mathbf{x}_{s}=\left\{\mathbf{r}_{\alpha}, \dot{\mathbf{r}}_{\alpha}\right\}$, the Hamiltonian microstates $\mathbf{y}_{s}=\left\{\mathbf{r}_{\alpha}, \mathbf{p}_{\alpha}\right\}$, possess no clear operational meaning. The field microstates are defined in terms of mode quaderatures within reciprocal space. As has been known for some time the classical treatment of the radiation field results in a divergent equilibrium free energy making the introduction of an ultraviolet cut-off necessary. When the field is in a thermal state the partition function $Z_{b}$ can be expressed as a product of single mode functions by assuming periodic field boundary conditions; $Z_{b}=\prod_{k} Z_{k}$. In the regime $\beta \omega \ll 1$, which constrains the validity of the classical treatment, the classical function $Z_{k}=1 / \beta \omega$ associated with the mode frequency $\omega=|\mathbf{k}|$ approximates the distribution $Z_{k}=1 /\left(1-e^{-\beta \omega}\right)$. This distribution yields a convergent free energy and is obtained from a quantum description of the field.

Using eq. (19) the rates of heat and work changes in the particle system are found to be

$$
\begin{aligned}
\dot{q} & =\int d^{3} x \mathbf{J} \cdot \mathbf{E}_{\mathrm{T}}=-\dot{E}_{b}, \\
\dot{w} & =\int d^{3} x \rho \dot{\phi}_{e}-\mathbf{J} \cdot \dot{\mathbf{A}}_{e} .
\end{aligned}
$$

The equality $\dot{q}=-\dot{E}_{b}$ can be obtained from Poynting's theorem, for the change in energy within a volume containing the particles by choosing the volume to be the whole space at the boundary of which the fields vanish.

Gauge freedom - The Lagrangian approach also sheds light on the issue of gauge freedom within the definition of work [29-39]. The authors [29] were the first to point out that the work defined by $\dot{w}=\dot{\lambda}_{t} \partial H / \partial \lambda_{t}$ is not unique due to the possibility of gauge freedom $H \rightarrow H+g(t)$ in the Hamiltonian. Here $g$ is an arbitrary time-dependent function. It has been suggested that when defined in terms of the Hamiltonian the quantity $-\beta^{-1} \ln \left(Z_{t} / Z_{0}\right)$ does not generally coincide with the system's free energy difference. However, eqs. (10) and (16) give gaugeinvariant definitions of work and free energy in terms of the energy $E_{s}$ directly. The resulting Jarzynski equality (15) is valid, independent of a possible freedom to choose the Hamiltonian.

For example, let us consider charged particles in an external field. The Lagrangian describing this situation is $L=L_{s}-\int d^{3} x[\rho \phi-\mathbf{J} \cdot \mathbf{A}]$ where $L_{s}$ is the total kinetic energy of the particles. Performing a gauge transformation of the external potentials $(\phi, \mathbf{A}) \rightarrow(\phi-\dot{\chi}, \mathbf{A}+\nabla \chi)$ leaves the Lorentz force equations unchanged. The interaction $L_{i}=L-L_{s}$ changes under the gauge transformation by the addition of a total time derivative; $L_{i} \rightarrow L_{i}-g(t)$ where $g(t):=-\sum_{\alpha} e_{\alpha} d \chi\left(\mathbf{r}_{\alpha}, t\right) / d t$. The canonical momenta therefore transform as $\mathbf{p}_{\alpha} \rightarrow$ $\mathbf{p}_{\alpha}-e_{\alpha} \nabla_{\alpha} \chi\left(\mathbf{r}_{\alpha}, t\right)$, and the Hamiltonian changes as $H \rightarrow H+g(t)$. However, $E_{s}=\sum_{\alpha} \dot{\mathbf{r}}_{\alpha} \cdot \partial L_{s} / \partial \dot{\mathbf{r}}_{\alpha}-L_{s}$ is manifestly invariant under the gauge transformation. The work, heat and free energy defined in terms of $E_{s}$ are gauge-invariant.

Conclusions - We have derived the first and second laws of thermodynamics using a Lagrangian approach rather than a Hamiltonian approach for certain important cases in which the two approaches differ. The Lagrangian formalism allows for an identification of the system and bath microstates such that the total energy is an extensive quantity. Although valid for any coupling strength the resulting thermodynamic theory is conceptually very simple. Heat and work components of the system's energy changes result from implicit and explicit time dependence respectively, and all thermodynamic entropies are defined in the same way as in information theory. A further advantage of the Lagrangian approach is that the microstates are manifestly gauge-invariant. This implies in particular, that no gauge-dependence issues arise within the definitions of work and free energy. As an example application of the formalism we have considered charged particles in the electromagnetic field. Unlike the Hamiltonian approach the Lagrangian approach yields a thermodynamic partition of the total energy which is consistent with the electrodynamic definition of transverse energy-momentum.

We have not discussed the quantisation of the theory in this letter. Formally quantisation can be carried out straightforwardly via the usual procedure of promoting variables to operators and replacing Poisson brackets with commutators. However, since the interaction is described through non-commutativity (with respect to the Poisson bracket) of the system and bath observables, upon quantisation the system and bath would not comprise conventional quantum subsystems. The system and bath must instead be viewed as dressed due to their in- 
teraction. A complete treatment of the quantum case is left for further work.

Acknowledgment. I thank Drs P. A. Knott, A. Nazir, T. Galla and D. Newman for useful discussions, and I thank Professor U. Seifert for helpful comments. I acknowledge financial support from the UK engineering and physical sciences research council.
[1] C. Jarzynski, Physical Review Letters 78, 2690 (1997), URL http://link.aps.org/doi/10.1103/ PhysRevLett.78.2690.

[2] C. Jarzynski, Journal of Statistical Mechanics: Theory and Experiment 2004, P09005 (2004), ISSN 17425468, URL http://stacks.iop.org/1742-5468/2004/ $i=09 / a=P 09005$.

[3] G. E. Crooks, Physical Review E 60, 2721 (1999), URL http://link.aps.org/doi/10.1103/PhysRevE. 60.2721.

[4] M. Campisi, P. Talkner, and P. Hnggi, Physical Review Letters 102, 210401 (2009), URL http://link. aps.org/doi/10.1103/PhysRevLett.102.210401.

[5] M. Esposito, U. Harbola, and S. Mukamel, Reviews of Modern Physics 81, 1665 (2009), URL http://link. aps.org/doi/10.1103/RevModPhys.81.1665.

[6] M. Campisi, P. Hnggi, and P. Talkner, Reviews of Modern Physics 83, 771 (2011), URL http://link.aps .org/ doi/10.1103/RevModPhys.83.771.

[7] J. Liphardt, S. Dumont, S. B. Smith, I. Tinoco, and C. Bustamante, Science 296, 1832 (2002), ISSN 00368075, 1095-9203, URL http://science.sciencemag. org/content/296/5574/1832.

[8] D. Collin, F. Ritort, C. Jarzynski, S. B. Smith, I. Tinoco, and C. Bustamante, Nature 437, 231 (2005), ISSN 00280836, URL http://www.nature.com/nature/journal/ v437/n7056/full/nature04061.html.

[9] F. Ritort, in Advances in Chemical Physics, edited by S. A. Rice (John Wiley \& Sons, Inc., 2007), pp. 31-123, ISBN 978-0-470-23808-0, URL http://onlinelibrary. wiley.com/doi/10.1002/9780470238080.ch2/summary.

[10] N. C. Harris, Y. Song, and C.-H. Kiang, Physical Review Letters 99, 068101 (2007), URL http://link.aps.org/ doi/10.1103/PhysRevLett.99.068101.

[11] A. N. Gupta, A. Vincent, K. Neupane, H. Yu, F. Wang, and M. T. Woodside, Nature Physics 7, 631 (2011), ISSN 1745-2473, URL http://www.nature.com/nphys/ journal/v7/n8/full/nphys2022.html.

[12] A. Alemany, A. Mossa, I. Junier, and F. Ritort, Nature Physics 8, 688 (2012), ISSN 17452473, URL http://www.nature.com/nphys/journal/ v8/n9/full/nphys2375.html.

[13] U. Seifert, Physical Review Letters 116, 020601 (2016), URL http://link.aps.org/doi/10.1103/ PhysRevLett.116.020601.

[14] R. Alicki, Journal of Physics A: Mathematical and General 12, L103 (1979), ISSN 0305-4470, URL http:// stacks. iop.org/0305-4470/12/i=5/a=007.

[15] H.-P. Breuer and F. Petruccione, The Theory of Open Quantum Systems (OUP Oxford, 2007), new ed ed., ISBN 0-19-921390-9.

[16] M. Esposito, K. Lindenberg, and C. V. d. Broeck, New Journal of Physics 12, 013013 (2010), ISSN 13672630, URL http://stacks.iop.org/1367-2630/12/i= $1 / \mathrm{a}=013013$.
[17] R. Kosloff, Entropy 15, 2100 (2013), URL http://www. mdpi.com/1099-4300/15/6/2100.

[18] A. E. Allahverdyan and T. M. Nieuwenhuizen, Physical Review Letters 85, 1799 (2000), URL http://link.aps . org/doi/10.1103/PhysRevLett. 85.1799.

[19] A. E. Allahverdyan and T. M. Nieuwenhuizen, Physical Review E 64, 056117 (2001), URL http://link.aps. org/doi/10.1103/PhysRevE.64.056117.

[20] H. Leff and A. F. Rex, eds., Maxwell's Demon 2 Entropy, Classical and Quantum Information, Computing (CRC Press, Bristol ; Philadelphia, 2002), 1st ed., ISBN 978-07503-0759-8.

[21] T. M. Nieuwenhuizen and A. E. Allahverdyan, in AIP Conference Proceedings (AIP Publishing, 2002), vol. 643, pp. 29-34, URL http://scitation.aip.org/content/ aip/proceeding/aipcp/10.1063/1.1523777.

[22] C. Hrhammer and H. Bttner, Journal of Physics A: Mathematical and General 38, 7325 (2005), ISSN 03054470, URL http://stacks.iop.org/0305-4470/38/i= $33 / a=008$

[23] J. G. Kirkwood, The Journal of Chemical Physics 3, 300 (1935), ISSN 0021-9606, 1089-7690, URL http://scitation.aip.org/content/aip/journal/ jcp/3/5/10.1063/1.1749657.

[24] H. Weimer, M. J. Henrich, F. Rempp, H. Schrder, and G. Mahler, EPL (Europhysics Letters) 83, 30008 (2008), ISSN 0295-5075, URL http://stacks.iop.org/ $0295-5075 / 83 / i=3 / a=30008$.

[25] H. Hossein-Nejad, E. J. OReilly, and A. Olaya-Castro, New Journal of Physics 17, 075014 (2015), ISSN 13672630, URL http://stacks.iop.org/1367-2630/17/i= $7 / a=075014$

[26] B. Roux and T. Simonson, Biophysical Chemistry $\mathbf{7 8}, 1$ (1999), ISSN 0301-4622, URL http://www.sciencedirect.com/science/article/ pii/S0301462298002269.

[27] M. F. Gelin and M. Thoss, Physical Review E 79, 051121 (2009), URL http://link.aps.org/doi/10. 1103/PhysRevE.79.051121.

[28] M. Esposito and C. V. d. Broeck, EPL (Europhysics Letters) 95, 40004 (2011), ISSN 0295-5075, URL http: //stacks. iop.org/0295-5075/95/i=4/a=40004.

[29] J. M. G. Vilar and J. M. Rubi, Physical Review Letters 100, 020601 (2008), URL http://link.aps.org/ doi/10.1103/PhysRevLett.100.020601.

[30] L. Peliti, Physical Review Letters 101, 098903 (2008), URL http://link.aps.org/doi/10.1103/ PhysRevLett.101.098903.

[31] J. Horowitz and C. Jarzynski, Physical Review Letters 101, 098901 (2008), URL http://link.aps.org/doi/ 10.1103/PhysRevLett.101.098901.

[32] J. M. G. Vilar and J. M. Rubi, Physical Review Letters 101, 098902 (2008), URL http://link.aps.org/ doi/10.1103/PhysRevLett.101.098902.

[33] J. M. G. Vilar and J. M. Rubi, Physical Review Let- 
ters 101, 098904 (2008), URL http://link.aps.org/ doi/10.1103/PhysRevLett.101.098904.

[34] L. Y. Chen, The Journal of Chemical Physics 129, 091101 (2008), ISSN 0021-9606, 1089-7690, URL http://scitation.aip.org/content/aip/journal/ jcp/129/9/10.1063/1.2978949.

[35] G. E. Crooks, The Journal of Chemical Physics 130, 107101 (2009), ISSN 0021-9606, 1089-7690, URL http://scitation.aip.org/content/aip/journal/ jcp/130/10/10.1063/1.3080751.

[36] L. Y. Chen, The Journal of Chemical Physics 130, 107102 (2009), ISSN 0021-9606, 1089-7690, URL http://scitation.aip.org/content/aip/journal/ jcp/130/10/10.1063/1.3080747.

[37] A. B. Adib, The Journal of Chemical Physics 130, 247101 (2009), ISSN 0021-9606, 1089-7690, URL http://scitation.aip.org/content/aip/journal/ jcp/130/24/10.1063/1.3158474.

[38] E. N. Zimanyi and R. J. Silbey, The Journal of Chemical Physics 130, 171102 (2009), ISSN 0021-9606, 10897690, URL http://scitation.aip.org/content/aip/ journal/jcp/130/17/10.1063/1.3132747.

[39] L. Peliti, Journal of Statistical Mechanics: Theory and Experiment 2008, P05002 (2008), ISSN 17425468, URL http://stacks.iop.org/1742-5468/2008/ $i=05 / a=P 05002$.

[40] A. V. Skorohod, Integration in Hilbert Space (Springer Berlin Heidelberg, Berlin, Heidelberg, 1974), ISBN 9783-642-65634-7 978-3-642-65632-3, URL http://link. springer.com/10.1007/978-3-642-65632-3.

[41] D. Janzing, P. Wocjan, R. Zeier, R. Geiss, and T. Beth,
International Journal of Theoretical Physics 39, 2717 (2000), ISSN 0020-7748, 1572-9575, URL http://link. springer.com/article/10.1023/A: 1026422630734.

[42] J. Oppenheim, M. Horodecki, P. Horodecki, and R. Horodecki, Physical Review Letters 89, 180402 (2002), URL http://link.aps.org/doi/10.1103/ PhysRevLett.89.180402.

[43] D. Jennings and T. Rudolph, Physical Review E 81, 061130 (2010), URL http://link.aps.org/doi/10. 1103/PhysRevE.81.061130.

[44] S. Jevtic, D. Jennings, and T. Rudolph, Physical Review Letters 108, 110403 (2012), URL http://link. aps.org/doi/10.1103/PhysRevLett.108.110403.

[45] F. G. S. L. Brandao, M. Horodecki, J. Oppenheim, J. M. Renes, and R. W. Spekkens, Physical Review Letters 111, 250404 (2013), URL http://link.aps.org/doi/ 10.1103/PhysRevLett.111.250404.

[46] F. Brandao, M. Horodecki, N. Ng, J. Oppenheim, and S. Wehner, Proceedings of the National Academy of Sciences 112, 3275 (2015), ISSN 0027-8424, 1091-6490, URL http://www . pnas .org/content/112/11/3275.

[47] R. Clausius, Annalen der Physik 169, 481 (1854), ISSN 1521-3889, URL http://onlinelibrary.wiley. com/doi/10.1002/andp.18541691202/abstract.

[48] H. Tasaki, arXiv:cond-mat/0009244 (2000), arXiv: condmat/0009244, URL http://arxiv.org/abs/cond-mat/ 0009244.

[49] C. Cohen-Tannoudji, J. Dupont-Roc, and G. Grynberg, Atom-photon interactions: basic processes and applications (Wiley, New York, 1992), ISBN 0-471-62556-6 9780-471-62556-8 0-471-29336-9 978-0-471-29336-1. 